\title{
Evaluation of Epworth Sleepiness Scale in patients with obstructive sleep apnea hypopnea syndrome
}

\section{Letícia Boari ${ }^{1}$, Carolina M. Cavalcanti ${ }^{2}$, Samantha R. F. D. Bannwart ${ }^{3}$, Oscimar B. Sofia4, José Eduardo L. Dolci ${ }^{5}$}

Key words: obstructive sleep apnea-hypopnea syndrome; Epworth Sleepiness Scale; polysomnography; apneahypopnea index.

\section{Summary}

\begin{abstract}
T
oday obstructive sleep apnea-hypopnea syndrome (OSAHS) is a public health issue, since it increases cardiovascular morbidity-mortality rate and the risk of car crashes. Overnight polysomnography is the gold standard for diagnosis and follow-up of affected patients. However, because the test is expensive, time-consuming and of difficult access, others methods have been proposed. Although the Epworth Sleepiness Scale (ESS) is subjective, the questionnaire is simple, easy to be applied and free of charge. Aim: to compare Epworth Sleepiness Scale scores and apnea-hypopnea index (AHI), measured by polysomnography, in patients diagnosed with OSAHS. Study Design: clinical retrospective study. Method: chart analysis of 66 patients complaining from snoring, who underwent surgery (uvulopalatopharyngoplasty with/without nasal surgery). ESS score and AHI were evaluated before and after surgery. Results: $78 \%$ of patients with normal AHI, scored $<10$ in the ESS and $65 \%$ of patients with severe AHI scored $>10$. There were no statistically significant results for groups presenting mild and moderate apnea. Conclusion: ESS can detect normal and severe levels of apnea, but is not able to detect mild and moderate levels. Therefore, ESS can be used in the follow-up of patients with OSAHS, however, it cannot replace polysomnography because it does not detect all levels of apnea.
\end{abstract}

\footnotetext{
${ }^{1}$ Master studies under course, Department of O torhinolaryngology, FCMSCSP, sponsored by CNPq.

${ }^{2}$ Resident Physician, Department of O torhinolaryngology, Santa Casa de Sao Paulo.

${ }^{3}$ Resident Physician, Department of O torhinolaryngology, Santa Casa de Sao Paulo.

${ }^{4}$ Master studies under course, Department of O torhinolaryngology, FCMSCSP, sponsored by CNPq. Craniomaxillofacial surgeon and O torhinolaryngologist, CEMA. ${ }^{5}$ Joint Professor, Department of O torhinolaryngology, FCMSCSP. Medical School, Santa Casa de Sao Paulo.

Address correspondence to: Rua dos Heliotrópios 133/94 Mirandópolis São Paulo SP 04049 -000 Fax: (55 11)5575-12 23 - E-mail: boari@osite.com.br

Study presented as poster at III Congresso Triológico de Otorrinolaringologia, held on October 08 - 11, 2003, in Rio de Janeiro. Article submited on July 29, 2004. Article accepted on March 30, 2004.
} 


\section{INTRODUCTION}

Many patients search for medial care to treat snoring, especially when it causes family/marriage issues. Excessive noise is the major complaint, but other signs also occur. Usually, complaints such as fatigue, daytime sleepiness, behavioral and cognitive dysfunction are not taken into account by the patient, who believes that such symptoms result from physical/ emotional stress and/ or aging. However, an appropriate work up is able to prove that snoring may be a symptom of a sleep disorder, obstructive sleep apnea-hypopnea syndrome (OSAHS). Estimates show that snoring prevalence in adults in the $5^{\text {th }}$ and $6^{\text {th }}$ decades of life is $40-60 \%$, and $90-95 \%$ of patients with OSAHS snore ${ }^{1}$.

This syndrome is currently considered a public health issue because it increases the risk of w ork-related and traffic accidents, as w ell as cardiovascular morbidity-mortality rate. Its prevalence varies according to the population. In the United States, estimates are of $4 \%$ in men and $2 \%$ in women between 30-60 years, especially in obese patients. However, these data are underestimated, and about $95 \%$ of the patients with sleep disorders are not diagnosed ${ }^{1,2}$. Haponik et al. ${ }^{3}$ showed in their study that none of the primary care physicians included sleep disorders in their differential diagnosis lists. On the other hand, when they became aware of the disease, $82 \%$ of the doctors started to investigate sleep disorders. Therefore, the health care provider, especially the otorhinolaryngologist, should be aware of this syndrome to diagnose and treat it promptly, preventing its severe consequences.

Currently, overnight polysomnography is the gold standard for diagnosis of OSAHS. Recordings of electroencephalogram, electrooculogram, electromyography, electrocardiogram, oxymetry, airflow and breathing effort provide important data to evaluate the severity of the disease. How ever, the test is expensive, time-consuming, of difficult access outside big cities and is not always well accepted by patients. ${ }^{4,5}$

Although subjective, the Epworth Sleepiness Scale may contribute to the analysis of signs and symptoms. It is easy to be applied, fast and free of charge. ${ }^{6}$

In summary, the purpose of our study was to evaluate Epworth Sleepiness Scale scores and apnea and hypopnea index $(\mathrm{AHI})$, measured by polysomnography, in patients diagnosed with OSAHS, as well as to compare these parameters.

\section{OBJECTIVE}

The purpose of our study was to evaluate pre and postoperative Epw orth Sleepiness Scale Scores and AHI in patients diagnosed with OSAHS, and compare these parameters.

\section{METHODS}

The study was conducted through chart analysis of patients complaining from snoring, from June 1999 to June 2003, at the Department of Otorhinolaryngology in Hospital CEMA. The inclusion criteria were adult men and women complaining from snoring, who underwent uvulopalatopharyngoplasty (UPPP). We used modified Fairbanks technique, with and without nasal approach (septoplasty and/ or partial inferior turbinectomy); pre and postoperative polysomnography was conducted and the questionnaire for sleep evaluation, the Epworth Sleepiness Scale (ESS) (Table 1), was ap plied before and after surgery. All charts contained complete physical examination performed by an otorhinolaryngologist.

The cases excluded from the study were: charts with incomplete data, patients presenting concurrent diseases (cardiopathy, thyroid disorders, diabetes mellitus and chronic lung disease), craniofacial dimorphism or retrognathia and patients who underwent UPPP in other departments. It is worth noting that the postoperative data were obtained, at least, 6 months after surgery. All surgeries were performed by the same surgical team.

Polysomnography, conducted in the Department of O torhinolaryngology or at Instituto Paulista do Sono, were in accordance with the minimal standards required, which were published on Atualização Otorrinolaringológica em Cirurgia do Ronco eApnéia do Sono (Otorhinolaryngological Update in Snoring Surgery and Sleep Apnea) by SBORL, $2002^{4}$.

We used the classification adopted by the American Academy of Sleep Medicine ${ }^{7}$ to categorize the level of dysfunction, as shown in Table 2.

Epw orth Sleepiness Scale was applied, the answers were given by the patient and the physician would only interfere in cases of doubt or misinterpretation.

Therefore, our study had 66 cases that met the criteria for inclusion. We considered 10 the normal limit of the Epw orth Sleepiness Scale, as stated in its original version. ${ }^{9}$

The statistical analysis was conducted by the test for similar proportions and Chi-square independence test. We found $95 \%$ confidence interval and $p=0.05$.

\section{RESULTS}

The studied group included 66 snoring patients who underwent surgery. UPPP without nasal approach (septoplasty and/or turbinectomy) was performed in only 8 patients, whereas the remaining patients underwent both surgeries. There were 6 women and 60 men, mean age was $42.47( \pm 10.7)$, ranging from 17 to 67 years old.

In the preoperative period, $72.7 \%$ (48) subjects had abnormal scores ( $>10)$ in ESS, whereas only $27.2 \%$ (18) had normal scores $(\leq 10)$. These scores ranged substantially 
Chart 1. Epworth Sleepiness Scale.

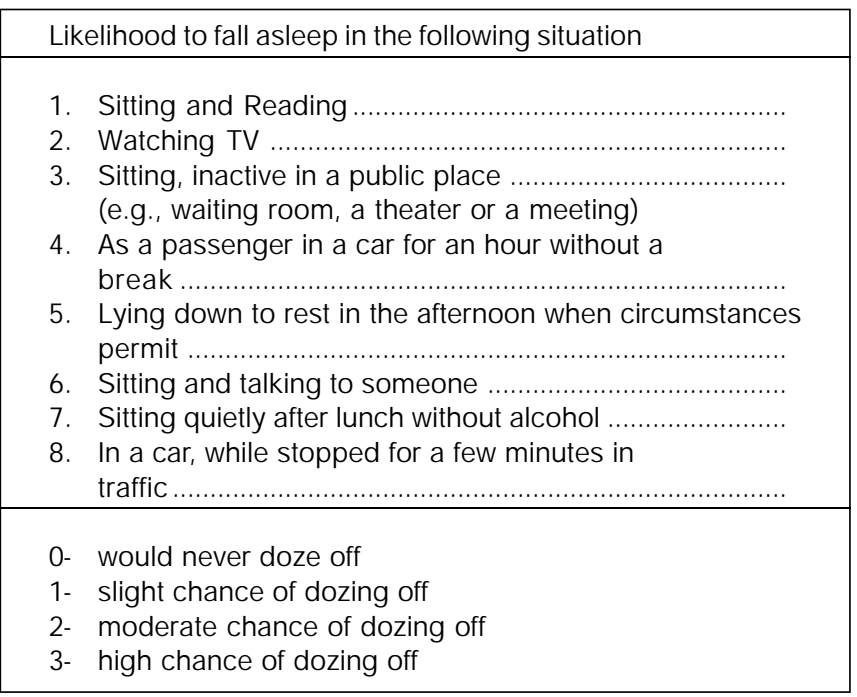

Chart 2. Classification of the severity level of OSAHS according to the American Academy of Sleeping Medicine -1997.

\begin{tabular}{|cc|}
\hline APNEA/HYPOPNEA INDEX & LEVEL \\
\hline$<5$ & NORMAL \\
$5-15$ & MILD \\
$15-30$ & MODERATE \\
$>30$ & SEVERE \\
\hline
\end{tabular}

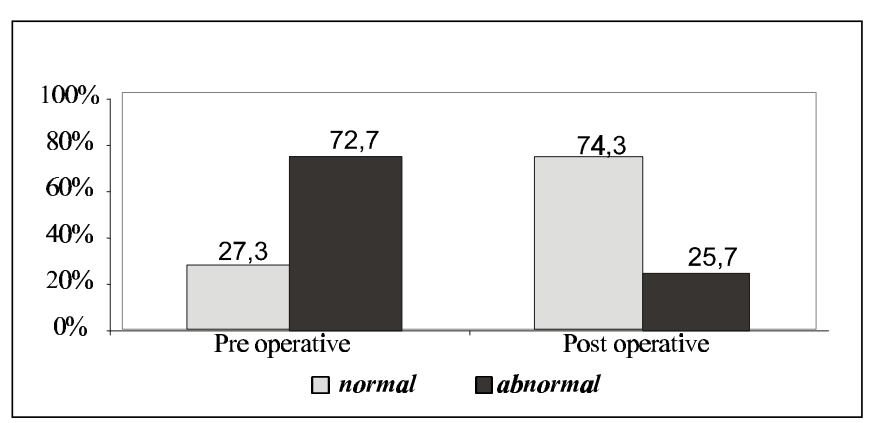

Chart 1. Pre and postoperative quantitative analysis of ESS.

Table 1. Pre and postoperative quantitative analysis of ESS.

\begin{tabular}{cccc}
\hline Epworth & Pre & Post & p-value \\
\hline Normal & $27.3 \%$ & $74.3 \%$ & $<0.001^{*}$ \\
Abnormal & $72.7 \%$ & $25.7 \%$ & $<0.001^{*}$ \\
\hline
\end{tabular}

during the postoperative period - $74.2 \%$ (49) had normal scores and $25.7 \%$ (17) had abnormal scores (Chart 1). These results showed statistically significant improvement $(p<0.001)$ in ESS scores after surgery, as shown in Table 1. Moreover, during the preoperative period, ESS normal average score was $6.6( \pm 2.5)$ and the abnormal average score was 16.0 ( \pm 3.4$)$. During postoperative examination, normal and abnormal average scores were $5.6( \pm 2.8)$ and 14.5 ( \pm 3.5$)$, respectively (Table 2 ).

During the preoperative period, $\mathrm{AHI}$ found in polysomnography was: $4.5 \%$ (3) normal; $28.78 \%$ (19) mild; $25.75 \%$ (17) moderate, and $40.90 \%$ (27) severe. During the postoperative period, we found $45.45 \%$ (30) normal, $21.21 \%$ (14) mild, $13.63 \%$ (9) moderate, and $19.69 \%$ (13) severe. Comparing the results before and after surgery, there was statistical significance in normal $\left(0.001^{*}\right)$ and severe $\left(0.005^{*}\right)$ scores, as shown in Table 3 and Chart 2.

The chi-square independence test was applied to check association between both variables studied. Because we had the purpose to analyze AHI and ESS scores in a given time point, without taking into account the reliability of methods, we mixed the preoperative and postoperative groups to obtain a most significant sample. Therefore, of the 33 patients with normal AHI, 26 (78.7\%) were normal according to the Epworth Sleepiness Scale and only 7 (21.\%) were abnormal. This result was statistically significant. As for mild and moderate levels, there was no correlation between parameters. Statistically significant results were also found in severe $\mathrm{AHI}$, and 26 (65\%) out of 40 patients had abnormal scores in the ESS - Table 4.

\section{DISCUSSION}

Obstructive Sleep Apnea and Hypopnea Syndrome has been carefully studied. In 1999, the American Academy of Sleep Medicine described this syndrome as recurrent episodes of total or partial upper airways obstruction, causing desaturation and sleep fragmentation. ${ }^{4}$ This is possibly associated with the frequent symptom of daytime sleepiness ${ }^{7}$. About $90 \%$ of patients affected with OSAHS show daytime sleepiness, whereas the remaining patients show changes only during overnight monitoring ${ }^{8}$.

It is generally accepted that overnight polysomnography is the gold standard for OSAHS diagnosis. According to Atualização Otorrinolaringológica em Cirurgia do Ronco e Apnéia do Son o by SBO RL, 2002, overnight recordings are the most significant ones, including electroencephalogram, electrooculogram, electromyography of submentonian and anterior tibial, electrocardiography, oxymetry, airflow determination and breathing effort ${ }^{4,5}$. However, the test is expensive and of difficult access.

Epw orth Sleepiness Scale was developed in 1991 by Dr. John W. Murray, an Australian physician. Ever since, it has been used globally, and it was translated into many 
Table 2. Pre and postoperative quantitative analysis of ESS.

\begin{tabular}{lcccc}
\hline Epworth & \multicolumn{2}{c}{ pre } & \multicolumn{2}{c}{ post } \\
& Normal & Abnormal & Normal & Abnormal \\
\hline Mean & 6.6 & 16.0 & 5.6 & 14.5 \\
Standard Deviation & 2.5 & 3.4 & 2.8 & 3.5 \\
Median & 6.0 & 15.0 & 5.5 & 13.0 \\
Minimum & 3.0 & 11.0 & 1.0 & 11.0 \\
Maximum & 10.0 & 23.0 & 10.0 & 22.0 \\
\hline
\end{tabular}

Table 3. Pre and postoperative analysis of AHI.

\begin{tabular}{lccc}
\hline AHI & Pre & Post & p-value \\
\hline Normal & $4.5 \%$ & $45.4 \%$ & $<0.001^{*}$ \\
Mild & $28.7 \%$ & $21.2 \%$ & 0.387 \\
Moderate & $25.7 \%$ & $13.6 \%$ & 0.076 \\
Severe & $40.9 \%$ & $19.6 \%$ & $0.005^{*}$ \\
\hline
\end{tabular}

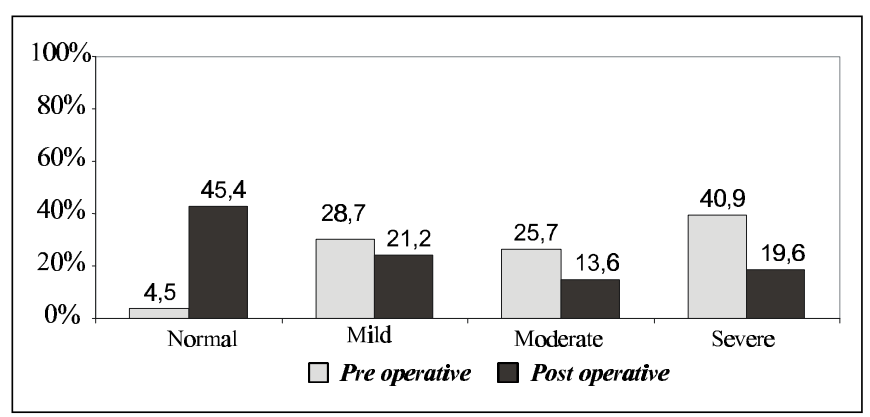

Chart 2. Pre and postoperative analysis of $\mathrm{AHI}$.

Table 4. Comparison between Epworth and $\mathrm{AHI}$, before and after surgery.

\begin{tabular}{llccc}
\hline Pre and Post & \multicolumn{2}{c}{ Epworth } & Total \\
& & Normal & Abnormal & \\
\hline AHI & Normal & 26 & 7 & 33 \\
& Mild & 17 & 16 & 33 \\
& Moderate & 10 & 16 & 26 \\
& Severe & 14 & 26 & 40 \\
\hline Total & 67 & 65 & 132 \\
\hline p-value $=0.001^{*}$
\end{tabular}

Table 5. Qualitative analysis of severe, moderate, mild and normal levels in association with Epworth Sleepiness Scale.

\begin{tabular}{lccccc}
\hline & IAH & & & & \\
ESS & severe & moderate & mild & normal & total \\
\hline mean & 12.83 & 11.66 & 10.37 & 7.71 & \\
standard & & & & & \\
deviation & 6.17 & 5.46 & 4.82 & 5.01 & \\
mode & 12 & 15 & 8 & 7 & \\
median & 12 & 13 & 10 & 7 & \\
minimum & 3 & 3 & 1 & 1 & \\
maximum & 23 & 21 & 23 & 22 & \\
\hline total & 41 & 27 & 32 & 32 & 132 \\
\hline
\end{tabular}

languages, such as German, Spanish and Japanese. Dr. Murray intended to quantify the likelihood of someone to fall asleep in 8 different common situations (see Chart 1). The scores range from 0 to 24, and 10 is the normal limit. According to the author, like any other Scale, the evaluation is not subjective but it is influenced by patient's reading and comprehension skills and honest answers. ${ }^{6}$

In his studies, Dr. Murray showed statistically significant results in ESS scores when comparing normal non-snoring subjects and snoring subjects. The average of the first group was $5.9 \pm 2.2(2-10), 10$ was considered the normal limit. ${ }^{9}$

Moreover, the Scale was able to separate primary snoring subjects from patients with OSAHS; the higher the ESS score, the more severe the condition. All patients with severe conditions scored over $10 .{ }^{6} \mathrm{H}$ ow ever, it is w orth noting that the severity level used by Dr. Murray was based on his judgment, where $\mathrm{AHI}<5$ was normal; 5 to 25 was mild; 25 to 50 was moderate, and $>50$ was severe; besides he only had 19 subjects in the severe group.

Our study showed that, separately, there was statistically significant increase of normal patients, in the pre and postoperative periods, considering both parameters, $\mathrm{AHI}$ and ESS. Moreover, corroborating the abovementioned study, there was statistically significant correlation between $\mathrm{AHI}$ and ESS when severe and normal groups were evaluated. Therefore, $65 \%$ of 40 severe patients scored over 10 in the ESS and $78.8 \%$ of 33 normal patients scored under 10. For mild and moderate groups, there was no statistically significant correlation. It is worth noting that, in our study, we put the preoperative and postoperative groups together to increase the sample of each subgroup. Therefore, another study separating such groups should be conducted in the future.

According to some authors, ESS cannot detect all levels of severity nor the frequency of apnea and hypopnea; therefore, ESS cannot replace polysomnography. ${ }^{8}$ Moreover, Nguyen et al ${ }^{10}$ showed that the Scale is not reliable and the score may vary up to 7 when the questionnaire is repeated within 6 months in the same group, with no interference but weight loss. However, we should consider that weight loss may have improved the condition and, consequently, interfered in ESS scores. O ur study could not evaluate test reliability because the patients underwent surgery and ESS was applied before and after surgery.

The normal limit of ESS has also been questioned. Douglas proposes $>12$ as normal based on a British study showing that $95 \%$ of normal people scored $11 . .^{8}$ If in our study we had considered 12 as normal, we would have still found the same statistically significant results for normal and severe groups.

Today, ESS helps to diagnose sleep disorders and recommend polysomnography. Our study contributed to attest that Epw orth Sleepiness Scale can be used to qualify, but not quantify, the level of disease. It can be used in the 
postoperative period of patients who are not willing to repeat polysomnography. It is worth saying that ESS should not replace polysomnography, but it can be an important tool in the follow-up period.

Further studies are needed to better characterize the normal limit and reliability, so that it can become more significant in practice.

\section{CONCLUSION}

We concluded that ESS shows correlation with $\mathrm{AHI}$ for severe and normal levels, but not for mild and moderate levels. Therefore, it can be used in the follow-up of the postoperative period, but should not replace polysomnography as a diagnostic tool. Other studies are required to evaluate reliability and the normal limitfor ESS, based on current data for severity of the condition.

\section{REFERENCES}

1. Bittencourt LR, Togeiro SMGP, Bagnato MC. Diagnóstico da Síndrome da apnéia e hipopnéia obstrutiva do sono. In: Stamm A. ed. Rinologia; 2002; São Paulo: Komedi; 2002: 103-11.
2. Mercado JC. Identifying Obstructive Sleep Apnea: A Highly Prevalent and Underdiagnosed Disease. Physician Assistant 2003; 27(2): 39-45.

3. Haponik EF, Frye AM, Richards B et al. Sleep history is neglected diagnostic information. J Gen Intern Med. 1996;11: 759-61.

4. Atualização Otorrinolaringológica em Cirurgia de Ronco e Apnéia. Revista Brasileira de Otorrinolaringologia set/out 2002; 68(5) supl.3.

5. Zonato Al, Bittencourt LR, Martinho FL, Júnior JFS, Gregório LC, Tufik S. Association of Systematic Head and Neck Physical Examination With Severity of Obstructive Sleep ApneaHypopnea Syndrome. Laryngoscope. 2003; 113(6):973-80.

6. Murray JW. Daytime Sleepiness, Snoring and Obstructive Sleep Apnea: The Epworth Sleepiness Scale. Chest 1993; 103(1): 30-6.

7. Sleep-Related Breathing Disorders in Adults: Recommendations for Syndrome Definition and Measurement techniques in Clinical Research- AASM Task Force. Sleep 1999; 22(5): 66789.

8. Douglas NJ. The Obstructive Sleep Apnea/Hypopnea syndrome. Practical Neurology 2003; 3(1): 22-9.

9. Murray JW. A New Method for Measuring Daytime Sleepiness: The Epworth Sleepiness Scale. Sleep 1991; 14: 540-5.

10. Nguyen AT, Palayew M, Baltazan M, Guillon S, Small D. Evaluation of the reproducibility of the Epworth Sleepiness Scale. Chest 2002; 122(4): 425 - 43 S. 